Editorial

\title{
Creating an Impact in the Global Research Surge
}

Dinesh Kadam ${ }^{1}$

\author{
${ }^{1}$ Department of Plastic and Reconstructive Surgery, A J Institute of \\ Medical Sciences and A J Hospital and Research Centre, Mangalore, \\ Karnataka, India
}

Indian J Plast Surg 2019;52:265-266

In the last decade, there has been a remarkable rise in the research across the world and middle and lower-income countries, accounting for the most substantial growth. ${ }^{1}$ According to the National Science Foundation (NSF) of the United States, India stands among the top three in the world ranking in fields of Science \& Engineering (S\&E), contributing $5.31 \%$ of the total research output in 2018, along with China (20.67\%) and the United States (16.54\%). ${ }^{2}$ With an impressive average annual growth of 10.73\% between 2008 and 2018, India is among the "fast-growing" in terms of research output, marching ahead of China and the United States. Further, NSF 2018 data shows, between 2003 and 2014, a massive 304\% increase in medical articles from India, which is again far ahead of the developed counties. However, the scientific world does not merely recognize the volume alone but by its impact on others by citations. The citation data provide indications of scientific impact or influence by counting how frequently other journal articles reference an article. The S\&E publication output in the top $1 \%$ of the cited publications from 2006 to 2016 shows doubling India's performance (from 0.33 to 0.70 ), however, remains lesser to the United States (1.88), European Union (1.30), China (1.12), and Japan (0.88). ${ }^{3}$ In this upward trend, there appears to be higher collaborative work with multiple authors and international coauthorship for the research. ${ }^{4}$ Contrary to this trend, the research publications in India are still published mainly by domestic authors with less than $20 \%$ international collaboration. ${ }^{5}$

\section{How to Improve the Visibility and the Impact?}

The objective of any publication is the dissemination of knowledge to reach the scientific community and make an impact. The impact is visible and measured with various metrics when the article receives citations. For this to happen, the article's visibility and discoverability on a search engine are paramount.

\section{Selecting Keywords and Title}

Quite often than not, we find keywords provided in the manuscript are of the author's subjective choice than appropriate words. So, what is appropriate and why is it important?

Keywords are phrases or words that are used for indexing an article. Appropriate title and right keywords will significantly impact the chances of getting picked up when searched. This improves not only readership but citation, particularly in systematic reviews that synthesize evidence on a subject.

Generally, journals ask for 6 to 10 keywords to represent the core research matter of an article. The selection of suitable keywords is frequently a neglected part of manuscript preparation and mostly arbitrary. There are some tools available to guide the authors to select the most frequent and appropriate words for the manuscript.

Medical Subject Headlines (MeSH): MeSH is a comprehensive controlled vocabulary for indexing journal articles in the life sciences and serves as a thesaurus that facilitates searching. ${ }^{6}$ Created and updated by the United States National Library of Medicine (NLM), it is used by the MEDLINE/PubMed article database, the NLM's catalog, and other NLM database. ${ }^{7}$ The MeSH vocabulary is regularly updated by subject specialists in various areas. Each year hundreds of new concepts are added, and thousands of modifications are made.

The NLM provides service for MeSH terms for an article by "MeSH on Demand" available through their website free of cost. ${ }^{8}$ It identifies and highlights words corresponding to $\mathrm{MeSH}$ vocabulary in the submitted text of the manuscript or an abstract. Multiple searches may be required as each upload is limited to 10,000 characters. This also lists similar articles from PubMed relevant to the submitted text. It
Address for correspondence

Dinesh Kadam, MS, DNB, MCh, Department of Plastic and

Reconstructive Surgery, A J Institute of Medical Sciences and A J Hospital and Research Centre, Mangalore, Karnataka 575004, India

(e-mail: drkadam@yahoo.co.in).
DOI https://doi.org/ 10.1055/s-0039-1701061 ISSN 0970-0358.
C2019 Association of Plastic

Surgeons of India
License terms

(우요 $\Theta$ 


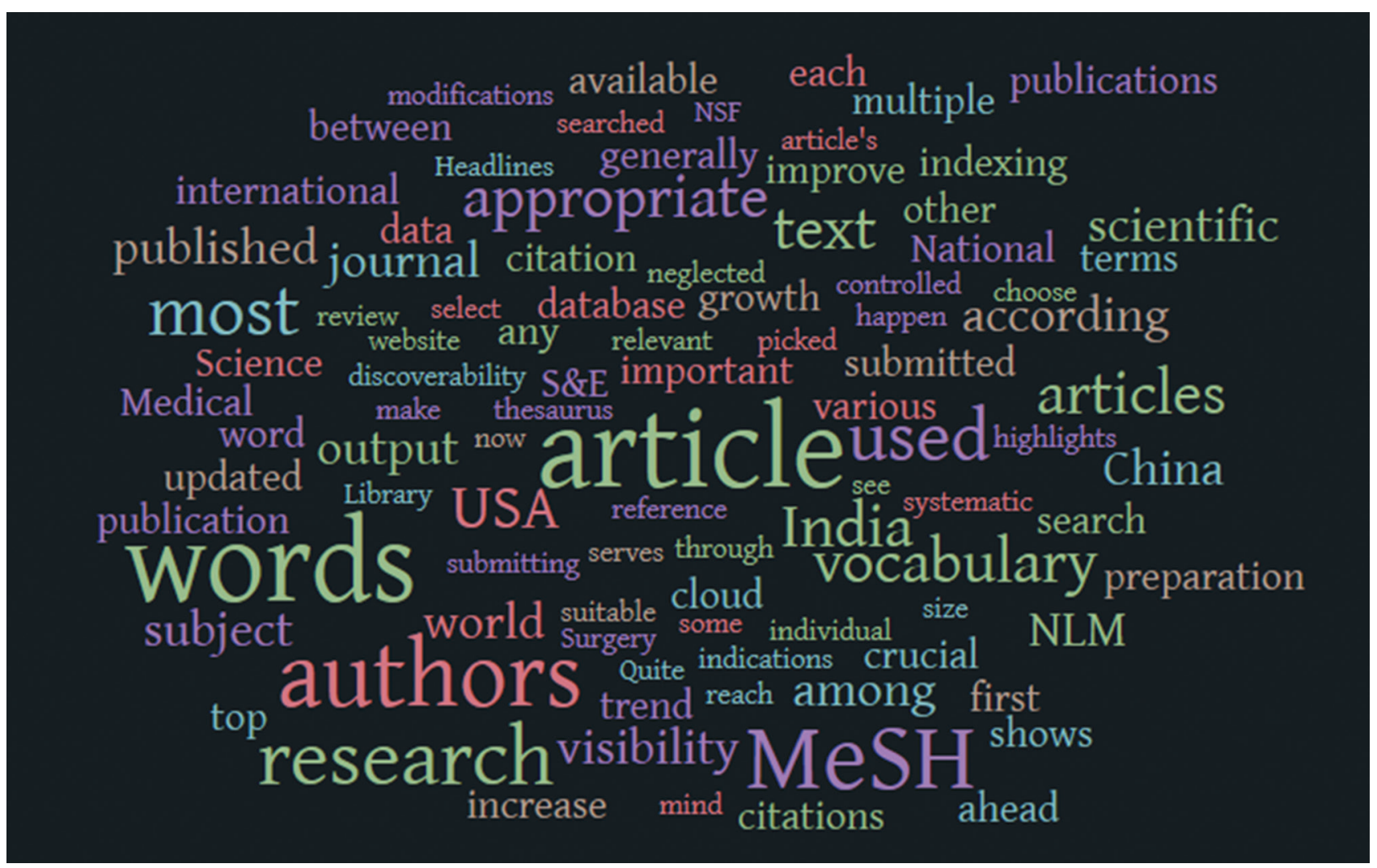

Fig. 1 Word cloud created from the website: https://worditout.com/word-cloud/create for this editorial text.

should be noted that the suggested MeSH vocabulary is machine-generated and does not reflect any human review.

Word cloud: The keywords generally are the most frequently used words of the article, but listing them manually is a laborious task. There are few websites to help in generating the most frequently used words in the text. ${ }^{9}$ A "word cloud" is presented as a cloud-like structure of words with varying sizes of the most frequently used words in the text. For example, a word cloud was created from the website:https://worditout.com/word-cloud/create for this editorial manuscript (-Fig. 1). Authors can choose words from the generated word cloud according to their size and relevancy. However, words generated not necessarily represent MeSH terms. A combination of word cloud and MeSH can help to boost the relevancy of words for the title and keywords. ${ }^{10}$ Most relevant keywords are taken from the list of words obtained with both searches. It is wise to frame the title after this exercise to represent the article appropriately. As far as possible keywords provided should not be repeated in the title as the title itself is strongly represented in the search.

To create an impact among the surge of research publication globally, we must provide every potential opportunity right from the stage of manuscript preparation. As the IJPS is marching ahead to receive its first impact factor, the role of authors cannot be overemphasized. We, therefore request authors to prepare manuscripts with greater attention for the high visibility and greater impact of their articles.

\section{References}

1 NSF. Available at: https://ncses.nsf.gov/pubs/nsb20206/publication-output-by-region-country-or-economy. Accessed November 5, 2019

2 Mccarthy $\mathrm{N}$. The countries leading the world in Scientific Publications [Infographic]. Available at: https://www. forbes.com/sites/niallmccarthy/2019/12/19/the-countriesleading-the-world-in-scientific-publications-infographic/ \#7e8607e31ec4. Accessed December 7, 2019

3 NSF. Available at: https://ncses.nsf.gov/pubs/nsb20206/ impact-of-published-research. Accessed December 7, 2019

4 Plume A, van Weijen D. Publish or perish? The rise of the fractional author. Available at: http://www.researchtrends.com/ issue-38-september-2014/publish-or-perish-the-rise-of-thefractional-author/. Accessed December 7, 2019

5 NSF. Available at: https://ncses.nsf.gov/pubs/nsb20206/international-collaboration. Accessed December 7, 2019

6 Medical Subjects Heading. Available at: https://en.wikipedia. org/wiki/Medical_Subject_Headings. Accessed December 7, 2019

7 NIH. Available at: https://www.nlm.nih.gov/mesh/meshhome. html. Accessed December 7, 2019

8 NIH. Available at: https://meshb.nlm.nih.gov/MeSHonDemand. Accessed December 9, 2019

9 WordItOut. Available at: https://worditout.com/word-cloud/ create. Accessed December 18, 2019

10 Mondal H, Mondal S, Mondal S. How to choose title and keywords for manuscript according to medical subject headings. Indian J Vasc Endovasc Surg 2018;5:141-144

\section{Conflict of Interest}

None declared. 